\title{
Identification of Prognostic Signature Based on the Copy Number Variation (CNV) and Expression in Acute Myeloid Leukemia
}

Changchun Niu ( $\square$ bright_star2000@163.com )

Chongqing General Hospital https://orcid.org/0000-0002-8847-7369

Di Wu

The University of Chicago Medical Center

Alexander J. Li

The University of Chicago Medical Center

Kevin H. Qin

The University of Chicago Medical Center

Daniel A. Hu

The University of Chicago Medical Center

\section{Eric J. Wang}

The University of Chicago Medical Centre

Andrew Blake Tucker

The University of Chicago Medical Centre

Fang He

The University of Chicago Medical Centre

Linjuan Huang

The University of Chicago Medical Centre

Hao Wang

The University of Chicago Medical Centre

Qing Liu

The University of Chicago Medical Centre

$\mathrm{Na} \mathrm{Ni}$

The University of Chicago Medical Centre

Deyao Shi

The University of Chicago Medical Centre

\section{Xia Zhao}

The University of Chicago Medical Centre

\section{Yafang Wan}

Chongqing General Hospital

Tian Li 
Chongqing General Hospital

\section{Tong-Chuan He}

The University of Chicago Medical Centre

\section{Pu Liao}

Chongqing General Hospital

\section{Research article}

Keywords: Acute myeloid leukemia, copy number variation, gene expression, prognosis

Posted Date: September 2nd, 2020

DOl: https://doi.org/10.21203/rs.3.rs-64413/v1

License: (c) (1) This work is licensed under a Creative Commons Attribution 4.0 International License. Read Full License 


\section{Abstract}

\section{Purpose}

Acute myeloid leukemia (AML) is caused by multiple genetic alterations in the hematopoietic progenitors, and molecular genetic analysis has provided useful information for AML diagnosis and prognosis. However, an integrative understanding about the prognosis value of specific copy number variation (CNV) and CNV-modulated gene expression has been limited.

\section{Methods}

We conducted an integrative analysis of CNV profiling and gene expression using data from the TARGET and TCGA AML cohorts. The CNV data from TCGA were analyzed using the GISTIC. CNV survival analysis and mRNA survival analysis were conducted with the Multivariate Cox proportional hazards regression model using R software with "survminer" and "survival" packages. KEGG cancer panel genes were extracted from the cancer related pathways from Kyoto Encyclopedia of Genes and Genomes (KEGG). The R package "circlize" was used for mapping the CNV genes to chromosomes.

\section{Results}

From this investigation, we observed distinct CNV patterns in the AML risk groups as well as the expression of 251 genes significantly modulated by CNV in both cohorts. There were $102 \mathrm{CNV}$ genes (located at 7q31-34, 16q24) associated with clinical outcomes in AML, which were identified in the TARGET cohort and validated in the TCGA cohort, three of which being miRNA genes (MIR29A, MIR183, MIR335) that overlapped with a KEGG cancer panel. Five genes were identified whose expressions were modulated by CNV and significantly associated with clinical outcomes, and among them, the deletion of $S E M A 4 D$ and $C B F B$ were found to potentially have protective effects against AML. Moreover, the distribution of $\mathrm{CNV}$ in these five $\mathrm{CNV}$-modulated genes was independent of the risk groups, which suggests that they are independent prognosis factors.

\section{Conclusion}

Overall, this study identified $102 \mathrm{CNV}$ genes and five CNV-modulated gene expressions that are crucial for developing new modes of prognosis evaluation and target therapy for AML.

\section{Introduction}

In 2008, it was newly reported for the first time that whole genome sequencing (WGS) was applied for an acute myeloid leukemia (AML) patient [1]. Today, the molecular analysis has provided large amounts of useful information for AML diagnosis, prognosis, and treatment[2]. The prognosis study provided the information of potential outcomes and survival rates, which is crucial for understanding the disease and improving its treatment[3]. Copy number variation (CNV) is a potential valuable prognosis marker. It is a type of structural variation that is larger than $50 \mathrm{bp}$, and involves unbalanced rearrangements that result 
in gains or losses of DNA content[4]. CNV drives genome evolution and alters the expressions of genes[5], which influence the progress of diseases such as cancers [6]. For example, in acute lymphoblastic leukemia (ALL) IKZFCNV was identified as a poor prognosis marker[7].

Though the prognosis and diagnosis of AML has shifted from histological analysis to a more comprehensive analysis such as genetic alteration[8], prognosis needs to be further improved, as about $60 \%$ of intermediate risk AML patients respond to therapy variously[9], and the AML patients with rarer abnormalities need be evaluated even more properly[10]. Moreover, the role of CNV functioning as a prognosis marker by modulating gene expression in AML remains elusive. Therefore, it is crucial to investigate the association between CNV and the prognosis of AML in order to better understand the genetic disorders of the disease. In turn, more novel, reliable predictions of outcomes for AML patients could arise.

To thoroughly understand the association between CNV and AML, we collected the CNV whole genome sequencing (WGS) data and gene expression data from the Therapeutically Applicable Research To Generate Effective Treatments (TARGET) AML cohort, and the CNV Array analysis data and gene expression data from The Cancer Genome Atlas (TCGA) consortium AML cohort. Through comprehensive analysis of CNVs and CNV-modulated gene expression-alterations, we show the essential CNV genes needed for the outcome and survival of AML patients.

\section{Materials And Methods}

\section{CNV data and gene expression data}

In this study, the TARGET and TCGA AML cohorts were used to analyze the prognostic roles of CNVs and CNV modulated gene expression. TARGET CNV (WGS data) and mRNA (mRNA and miRNA sequencing) data were downloaded from target-data.nci.nih.gov. The clinical data, including patient identification, gender, risk group, age, overall survival time and vital status, were downloaded and pre-processed. The CNV data (Affymetrix Genome-Wide SNP array 6.0 data), mRNA data (mRNA and miRNA sequencing), and clinical data of the TCGA AML cohort were downloaded from gdac.broadinstitute.org. All data used in the study were provided by TARGET and TCGA, therefore ethics approval and written informed consent were not required.

\section{Database preparation}

To reduce the influence of different synonyms of genes, the gene names were transferred to "symbol" based on the file "gene_info.gz" downloaded from ftp.ncbi.nlm.nih.gov.

KEGG cancer panel genes were extracted from the cancer related pathways using an API from https://www.genome.jp/kegg/, and 483 genes were included in the KEGG cancer panel. 


\section{Statistical analysis}

\subsection{The data from the primary blood derived cancer groups and solid normal tissue groups}

In TCGA were analyzed separately, using the GISTIC (version 2.0.23) downloaded from ftp.broadinstitute.org, and parameters were setup as TCGA suggested: the threshold for copy number amplifications was 0.1 , the threshold for copy number deletions was 0.1 , the maximum number of segments was 2000, and the significance threshold for q-values was 0.25 (https://docs.gdc.cancer.gov/Data/Bioinformatics_Pipelines/CNV_Pipeline/).

\subsection{CNV survival analysis}

The Multivariate Cox proportional hazards regression model (Cox model) were used for identifying survival-related CNVs in AML patients.

The R package "circlize" was used for mapping the CNV genes to chromosomes.

In TARGET cohorts, 193 patients (96 females and 97 males, median age 9 years) who had integrated CNV data (WGS data) and clinical data were included for survival analysis. 191 patients (87 females and 104 males, median age 58 years) who had integrated CNV data and clinical data were included for validating the survival analysis result from TARGET cohorts.

We removed those CNVs occurring in less than 4 samples. For every gene, patients were divided into two groups, named "normal" or "CNV," and analyzed with the Cox model adjusted for gender and age. The $p$ values were adjusted with "HDR" and the Kaplan-Meier curve was used for visualizing the survival. We performed all analyses using R software with "survminer" and "survival" packages.

\section{3 mRNA survival analysis}

The expressions of mRNA (RPKM) were log2-transformed. In TARGET cohorts, 294 patients (the recurring patients were excluded) who had integrated mRNA sequencing data and clinical data were included and analyzed with multivariate Cox proportional hazards regression models adjusted for gender and age, and univariate Cox proportional hazards regression models for gender or age. We used R "survminer" and "survival" packages to perform this analysis, and "HDR" to adjust the $p$ values afterwards. The gene expression data from the 294 TARGET AML cohort (137 females and 157 males, median age 10 years) were used for exploring the relationship between gene expression and survival. For statistical tests, $p<$ 0.05 , and adjusted $p<0.05$ were considered significant. In the TCGA AML cohorts, 179 patients (84 females and 95 males, median age 58 years) who had integrated CNV data and clinical data were included for validating the survival analysis results from the TARGET cohorts. 


\subsection{Integrative analysis of gene expression and CNV data}

Integrative analysis of gene expression and CNV was performed on 156 patients in the TARGET cohort and 171 patients in the TCGA cohort. Gene expression was compared between patient groups with different CNV statuses using the Kruskal test or Wilcox test. The $p$ values were adjusted with "HDR," and $p$ $<0.05$ and adjusted $p<0.2$ ("HDR") was considered to be statistically significant.

\section{VENN graphic presentations}

VENN diagrams were plotted with the R package "VennDiagram".

\section{Results}

\section{Distribution of the CNV}

The CNV data (WGS) from 193 TARGET AML cohort patients ( 96 females and 97 males, median age 9 years), and the CNV data (SNP array) from 191 TCGA cohort patients (87 females and 104 males, median age 58 years) were used to investigate the distribution of CNV in AML.

The results of the TARGET AML cohort showed that the distribution of CNV in 99.6\% (17507/17586) of said genes were below $6 \%$; however, the other $35 \mathrm{CNV}$ genes were above $90 \%$. By contrast, the distribution of CNVs in the TCGA cohort were at most $14.1 \%$ (Fig 1A).

Consistent with a previous report[9], this study showed that CNVs were not randomly distributed in the chromosomes. Amplifications were more frequent in chromosomes 1,8,19, 21, and 22 of the TARGET cohort and TCGA cohorts, and deletions were more frequent in chromosomes 7,16 , and X. There were few CNVs in the normal tissues compared to the AML samples (Fig1 B).

The results also showed distinct CNV distribution patterns in three risk groups in the TARGET cohort. CNVs occurred most frequently in the standard risk group; in the high risk groups, there were more frequent amplifications in chromosome 7 and more frequent deletions in chromosome 19; deletions in chromosome 7 frequently occurred in the standard and low risk groups; amplification in chromosome $\mathrm{X}$ mainly occurred in the standard group, and deletions in chromosome $\mathrm{X}$ mainly occurred in the low risk group.

\section{Integrative analysis of gene expressions in concordance with CNV}

It has been demonstrated that modulating gene expression is one of the most important ways for CNV to play diversified roles in diseases[11-14]. In this study, the results showed that 5022 gene expressions in TCGA were significantly modulated by CNV $(p<0.05$, adjusted $p<0.2)$ and 577 genes in TARGET were 
significantly associated with CNV ( $p<0.05$, adjusted $p<0.2)$. 251 genes overlapped in both cohorts (supplementary table 1), and among them 52.5\% (132/251) gene-specific CNVs were located in chromosome 19 (19q13, 19p13) (Fig 2A), and four genes overlapped with the KEGG cancer panel, including KEAP1, SIRT6, RHEB and DNMT1 (Fig 2B).

\section{Prognostic value of CNV in AML}

To further explore the prognostic values of CNVs, a Multivariate Cox proportional hazards regression model analysis was performed on the TARGET cohort, adjusted with age and gender $(p<0.05$, adjusted $p$ $<0.05$ ). There were $758 \mathrm{CNV}$ genes found to be significantly associated with patient survival. Among them, 102 CNV genes overlapped with the TCGA AML cohorts $(p<0.05)$ (supplementary table 2), including 7 IncRNA and 10 miRNA genes, implicating potential gene regulating capabilities.

Moreover, the CNV genes with the largest probability of high risk were located at chromosome 7 and 16 (7q31,7q32, 7q33, 7q34, 16q24.1) (Fig 3A). In addition, it was observed that 7.8\% (8/102) of these CNVs modulated gene expression in the TARGET cohort and $52.0 \%$ (53/102) of these CNVs modulated gene expression in the TCGA cohort respectively (Fig 3C).

We also compared these 102 genes with the KEGG cancer panel and found that three miRNA genes (MIR29A, MIR183, MIR335) overlapped with said panel (Fig 2B). COX model analyzing adjusted for age and gender additionally showed that MIR335 expression (miRNA sequencing data) was associated with survival in the TARGET cohort $(\mathrm{n}=300, p=0.015$, HR: 0.911 95\%:0.844-0.982, Fig 3DE).

\section{Identification of CNV-modulated genes expressions associated with survival}

The results showed that 2058 gene expressions were associated with survival in the TARGET AML cohort, 268 of which were validated in the TCGA cohort. Among these 268 genes were 7 CNV-regulated genes associated with survival in the TARGET cohort, and $87 \mathrm{CNV}$-regulated genes associated with survival in the TCGA AML cohort (Fig 4A). Furthermore, there were 5 genes in both cohorts whose expressions were associated with survival and also overlapped with concordant CNV directions, including CBFB (16q22), CHAF1B (21q22), DNMT1 (19p13), SAE1 (19q13) and SEMA4D (9q22) (Fig4 B). The effects of CNV on the expression of these five genes were shown in Fig4 C-L, and deletions of CBFB and SEMA4D downregulated the gene expressions, which implicated that CNVs of $C B F B$ and $S E M A 4 D$ may be protective, and amplification of $C H A F 1 B, S A E 1$, and $D N M T 1$ upregulated the gene expression.

Additionally, these CNVs were independent from the risk groups; in the TARGET cohort, they occurred more frequently in standard-risk patients, and in the TCGA cohort, they occurred more frequently in highrisk patients (Fig4 M). 


\section{Discussion}

Using a large number of subjects from the TARGET and TCGA AML cohorts, this study demonstrated not only the association between many gene-specific SNVs and AML prognosis but also provided valuable potential AML prognosis markers. Our study showed that the CNVs occurred with distinct patterns in AML risk groups; 251 genes whose expressions were modulated by CNV were identified in both cohorts, and among them were 4 genes that overlapped with the KEGG cancer panel. Additionally, $102 \mathrm{CNV}$ genes directly associated with the survival of AML were identified and located on chromosomes 7 and 16, and among them there were three miRNA genes that overlapped with the KEGG cancer panel; we also identified the five gene expressions modulated by CNV that have vital effects on the survival of subjects, and had no obvious relationship with risk groups in the two cohorts (Figure 5). The identification of these CNVs is crucial for developing new modes of prognosis evaluation and target therapy.

The array data were used on CNV analysis in previous AML studies [9, 15-17]. In this study, we used GWS data from TARGET to discover valuable CNV markers and validated them with the array data from TCGA. The CNV in the TARGET cohort showed a different distribution pattern than the TCGA cohort, which may be due to different approaches. A previous study indicated that GWS is the most powerful approach because of its high sensitivity and ability to provide accurate breakpoint information[18], whereas the resolution capacity of Array is limited[4]. In relation to the distribution of CNV frequency in the chromosomes, our study showed more frequent amplifications on chromosomes 8, 11 and 21, and more frequent deletions on chromosome 7, which is in consistent with 0 Nibourel et al's study[9]. Comparing the TCGA cohort with the normal tissues of AML subjects, CNVs occurred in AML tissues more frequently, supporting the crucial role of CNVs on cancer[19]. Distinct distribution patterns of CNV in risk-groups suggests that CNV is a potential risk stratification marker, which needs to be further studied and validated.

Modulating gene expression is the major approach for CNVs to play biological roles. In this study, it was observed that a large part of gene expressions $(28.5 \%$ genes (5022/17627) in the TCGA cohort, $7.4 \%$ genes (577/7839) in TARGET cohort) were modulated by CNV. And there were 251 overlapping genes in both cohorts, and the distribution of these genes in the chromosomes showed a similar pattern with the distribution of CNV, suggesting that CNV modulating expression is widespread in CNV. The different percentages of modulating in the two cohorts may be due to different CNV analysis approaches used.

Through survival analysis using the COX model, this study identified the $102 \mathrm{CNV}$ genes that locate at 7q31,7q32, 7q33, 7q34, and 16q24.1. Deletion in 7q33-34 has been previously reported[15]. Moreover, these 102 CNV genes included 7 IncRNA and 10 miRNA genes, which implicated potential gene regulating capability, as IncRNA and miRNA function as master regulators of gene expression[20, 21]. Furthermore, three miRNA genes (MIR29A, MIR183, MIR335) overlapped with cancer (KEGG cancer). It has been reported that miR-29a is a key epigenetic regulator and has crucial roles in cancers including AML[22], and that miR-335 is involved in diverse cancers including AML[23], and our result indicated that the high expression level of miR-335 is a protective factor. 
This study also identified 5 CNV genes that, through regulating gene expression, indirectly affected the outcomes of patients; among them, deletions of CBFB and SEMA4D induced down-regulating expression that may be protective. Moreover, these CNV distributions were independent from risk groups, which suggests that they may be independent prognostic markers. Previous studies have shown that some of them were associated with AML. CBFB forms fusion genes $C B F B / M Y H 11$, which block differentiation in AML[24], but the deletion of $C B F B$ usually was accompanied with the deletion of $M Y H 11$, and the deletion of $M Y H 11 / C B F B$ had no negative effect on the prognosis[25]. Our findings suggested deletion-induced downregulating $C B F B$ expression has positive effects on the prognosis. Elevated expressions of $O C H A F 1 B$ were observed in leukemia and are critical for leukemogenesis and maintaining the undifferentiated state of leukemia cells, and heterozygous deletions of Chaf $1 b$ in mice block leukemogenesis[26], and the results of this study showed that in both cohorts, CNV amplification of OCHAF1B led to expression upregulations and a negative prognosis. DNMT1, a member of DNA methyltransferases, was up-regulated and considered to be a potential oncogene in AML through downregulating $\mathrm{p} 15$ expression, and our result showed that $\mathrm{CNV}$-modulated upregulating DNMT1 expressions may lead to bad outcomes in AML patients. SAE1 is a key molecule in the small ubiquitin (Ub)-like modifier (SUMO) pathway, and promyelocytic leukemia protein (PML) and fusion protein PML-RARa (retinoic acid receptor-a) fusion oncoprotein are substrates of the SUMO pathway[27], but no direct association between SAE1 and AML was reported, and our study found that upregulated SAE1 expression derived from CNV amplifications were associated with poor outcomes. SEMA4D was considered a good predictor for worse clinical outcome[28], and our findings showed that deletion of SEMA4D induced downregulated expression was protective.

\section{Conclusion}

In conclusion, our study identified 102 CNV genes (7q31-34, 16q24) associated with a higher risk of worse outcomes, $5 \mathrm{CNV}$-modulating gene expressions associated with the risk of the patients' outcomes, and found that deletion of SEMA4D and CBFB might have protective effects.

\section{Declarations}

\section{Funding}

This work was supported by the National Natural Science Foundation of China (81702075) and the Natural Science Foundation of Chongqing (cstc2019jcyj-msxmX0276), China.

\section{Competing interests/Competing interests}

The authors declare no competing conflicts of interest.

\section{Ethics approval}


All procedures performed in studies involving human participants were in accordance with the ethical standards of the institutional and/or national research committee and with the 1964 Helsinki declaration and its later amendments or comparable ethical standards.

\section{Consent to participate}

Not applicable.

\section{Consent for publication}

The authors declare the consent for publication.

\section{Availability of data and material}

The dataset supporting the conclusions of this article is included within the article and its additional files.

\section{Code availability}

Not applicable.

\section{Authors' contributions}

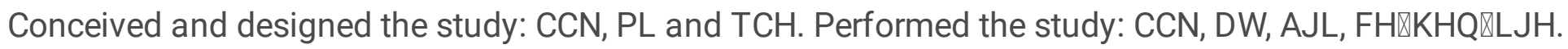
Analyzed the data: CCN, DAH, EJW, ABT, HW, QL. Wrote the paper: CCN, NN, DYS, XZ, WYF and TL. All authors read and approved the final manuscript.

\section{Acknowledgments}

The authors thank Therapeutically Applicable Research To Generate Effective Treatments (TARGET) and The Cancer Genome Atlas (TCGA) for providing the data. The reported work was supported in part by research grants from the National Natural Science Foundation of China (81702075) and the Natural Science Foundation of Chongqing, China (cstc2019jcyj-msxmX0276).

\section{References}

1. Ley TJ, Mardis ER, Ding L, Fulton B, McLellan MD, Chen K, Dooling D, Dunford-Shore BH, McGrath S, Hickenbotham $\mathrm{M}$, et al. DNA sequencing of a cytogenetically normal acute myeloid leukaemia genome. Nature. 2008;456(7218):66-72. 
2. Cai SF, Levine RL. Genetic and epigenetic determinants of AML pathogenesis. Semin Hematol. 2019;56(2):84-9.

3. Wee Y, Liu Y, Lu J, Li X, Zhao M. Identification of novel prognosis-related genes associated with cancer using integrative network analysis. Scientific reports. 2018;8(1):3233.

4. Zarrei M, MacDonald JR, Merico D, Scherer SW. A copy number variation map of the human genome. Nature reviews Genetics. 2015;16(3):172-83.

5. Liang L, Fang JY, Xu J. Gastric cancer and gene copy number variation: emerging cancer drivers for targeted therapy. Oncogene. 2016;35(12):1475-82.

6. Lauer S, Gresham D. An evolving view of copy number variants. Current genetics. 2019;65(6):128795.

7. Medeiros BC: Deletion of IKZF1 and prognosis in acute lymphoblastic leukemia. The New England journal of medicine 2009, 360(17):1787; author reply 1787-1788.

8. Arber DA. The 2016 WHO classification of acute myeloid leukemia: What the practicing clinician needs to know. Semin Hematol. 2019;56(2):90-5.

9. Nibourel O, Guihard S, Roumier C, Pottier N, Terre C, Paquet A, Peyrouze P, Geffroy S, Quentin S, Alberdi $A$, et al. Copy-number analysis identified new prognostic marker in acute myeloid leukemia. Leukemia. 2017;31(3):555-64.

10. Grimwade D, Hills RK, Moorman AV, Walker H, Chatters S, Goldstone AH, Wheatley K, Harrison CJ, Burnett AK. Refinement of cytogenetic classification in acute myeloid leukemia: determination of prognostic significance of rare recurring chromosomal abnormalities among 5876 younger adult patients treated in the United Kingdom Medical Research Council trials. Blood. 2010;116(3):354-65.

11. Berg KCG, Sveen A, Høland M, Alagaratnam S, Berg M, Danielsen SA, Nesbakken A, Søreide K, Lothe RA. Gene expression profiles of CMS2-epithelial/canonical colorectal cancers are largely driven by DNA copy number gains. Oncogene. 2019;38(33):6109-22.

12. Zhou B, Guo R. Integrative Analysis of Genomic and Clinical Data Reveals Intrinsic Characteristics of Bladder Urothelial Carcinoma Progression. Genes 2019, 10(6).

13. Henrichsen $\mathrm{CN}$, Chaignat $\mathrm{E}$, Reymond A. Copy number variants, diseases and gene expression. Human molecular genetics. 2009;18(R1):R1-8.

14. Shao X, Lv N, Liao J, Long J, Xue R, Ai N, Xu D, Fan X. Copy number variation is highly correlated with differential gene expression: a pan-cancer study. BMC Med Genet. 2019;20(1):175.

15. Kühn MW, Radtke I, Bullinger L, Goorha S, Cheng J, Edelmann J, Gohlke J, Su X, Paschka P, Pounds S, et al. High-resolution genomic profiling of adult and pediatric core-binding factor acute myeloid leukemia reveals new recurrent genomic alterations. Blood. 2012;119(10):e67-75.

16. Vujkovic M, Attiyeh EF, Ries RE, Goodman EK, Ding Y, Kavcic M, Alonzo TA, Wang YC, Gerbing RB, Sung $L$, et al. Genomic architecture and treatment outcome in pediatric acute myeloid leukemia: a Children's Oncology Group report. Blood. 2017;129(23):3051-8. 
17. Radtke I, Mullighan CG, Ishii M, Su X, Cheng J, Ma J, Ganti R, Cai Z, Goorha S, Pounds SB, et al. Genomic analysis reveals few genetic alterations in pediatric acute myeloid leukemia. Proc Natl Acad Sci USA. 2009;106(31):12944-9.

18. Liu B, Morrison CD, Johnson CS, Trump DL, Qin M, Conroy JC, Wang J, Liu S. Computational methods for detecting copy number variations in cancer genome using next generation sequencing: principles and challenges. Oncotarget. 2013;4(11):1868-81.

19. Shlien A, Malkin D. Copy number variations and cancer. Genome medicine. 2009;1(6):62.

20. Peng WX, Koirala P, Mo YY. LncRNA-mediated regulation of cell signaling in cancer. Oncogene. 2017;36(41):5661-7.

21. Bartel DP. MicroRNAs: target recognition and regulatory functions. Cell. 2009;136(2):215-33.

22. Gado MM, Mousa NO, Badawy MA, El Taweel MA, Osman A. Assessment of the Diagnostic Potential of miR-29a-3p and miR-92a-3p as Circulatory Biomarkers in Acute Myeloid Leukemia. Asian Pacific journal of cancer prevention: APJCP. 2019;20(12):3625-33.

23. Zhou JD, Li XX, Zhang TJ, Xu ZJ, Zhang ZH, Gu Y, Wen XM, Zhang W, Ji RB, Deng ZQ, et al. MicroRNA-335/ID4 dysregulation predicts clinical outcome and facilitates leukemogenesis by activating PI3K/Akt signaling pathway in acute myeloid leukemia. Aging. 2019;11(10):3376-91.

24. Opatz S, Bamopoulos SA, Metzeler KH, Herold T, Ksienzyk B, Bräundl K, Tschuri S, Vosberg S, Konstandin NP, Wang C, et al. The clinical mutatome of core binding factor leukemia. Leukemia. 2020;34(6):1553-62.

25. Lv L, Yu J, Qi Z: Acute myeloid leukemia with inv(16)(p13.1q22) and deletion of the $5^{\prime} \mathrm{MYH11/3}$ 'CBFB gene fusion: a report of two cases and literature review. Molecular Cytogenetics 2020, 13(1):4.

26. Volk A, Liang K, Suraneni P, Li X, Zhao J, Bulic M, Marshall S, Pulakanti K, Malinge S, Taub J, et al. A CHAF1B-Dependent Molecular Switch in Hematopoiesis and Leukemia Pathogenesis. Cancer cell. 2018;34(5):707-23.e707.

27. Seeler JS, Dejean A. SUMO and the robustness of cancer. Nature reviews Cancer. 2017;17(3):184-97.

28. de Boer B, Prick J, Pruis MG, Keane P, Imperato MR, Jaques J, Brouwers-Vos AZ, Hogeling SM, Woolthuis CM, Nijk MT, et al. Prospective Isolation and Characterization of Genetically and Functionally Distinct AML Subclones. Cancer cell. 2018;34(4):674-89.e678.

\section{Figures}



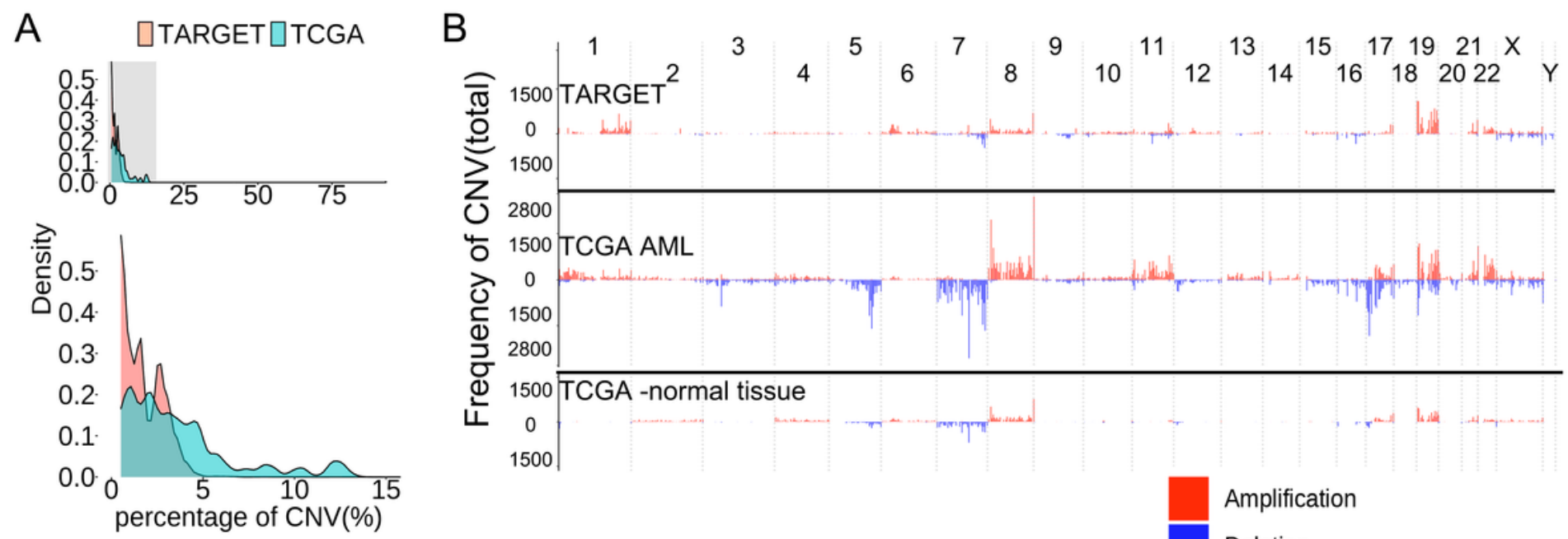

Amplification

Deletion

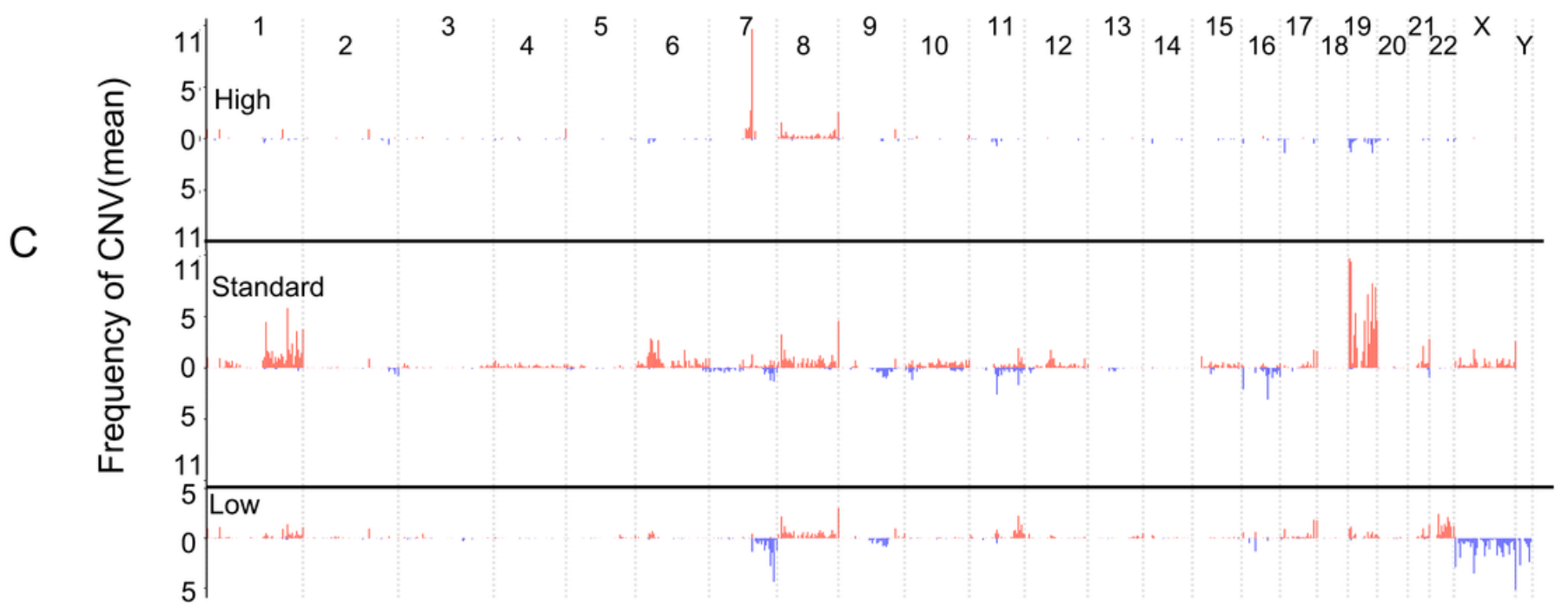

\section{Figure 1}

Genome-wide distribution of CNV specific genes in two AML cohorts. (a) Distribution of CNV percentages in the TARGET AML cohort ( $n=193)$, TCGA AML cohort $(n=191)$ and TCGA-normal tissue $(n=189)$. (b) Frequency of cumulative CNV counts (total CNV counts of all patients) in chromosome map locations. (c) Frequency of cumulative CNV counts (mean CNV counts of every patient) in chromosome map locations. 
A

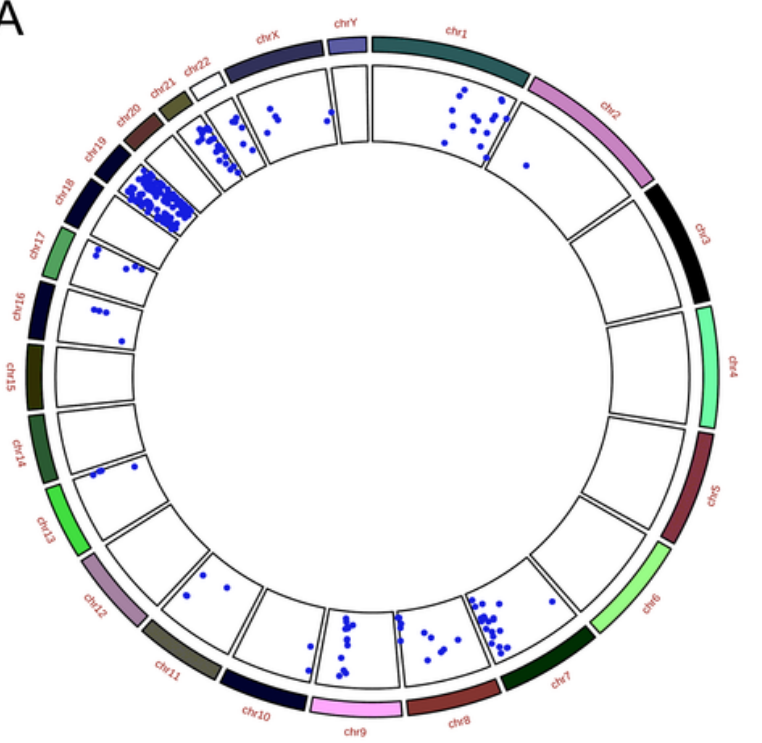

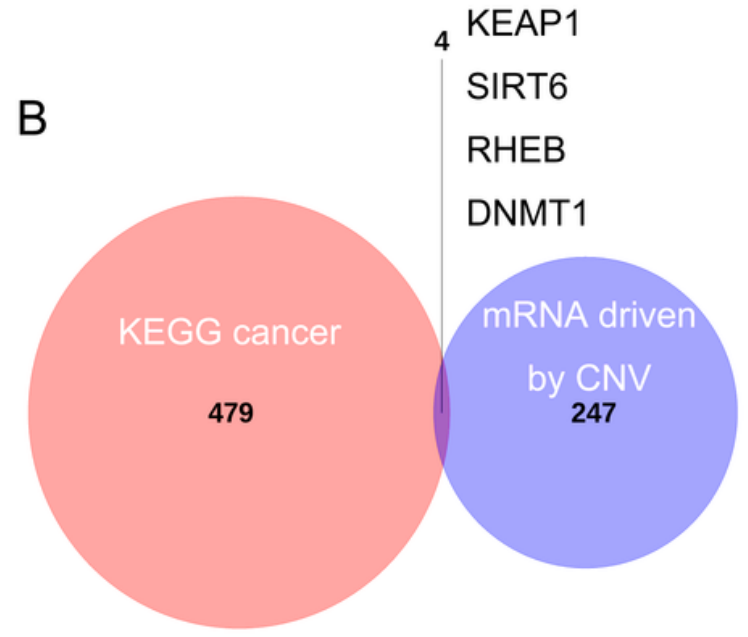

\section{Figure 2}

Distribution of genes modulated by CNV. (a) The distribution in chromosome map locations of the genes $(n=251)$ whose expressions were significantly modulated by CNV in both cohorts. (b) Venn diagram showed the relationship of the genes modulated by CNV and the KEGG cancer panel. 


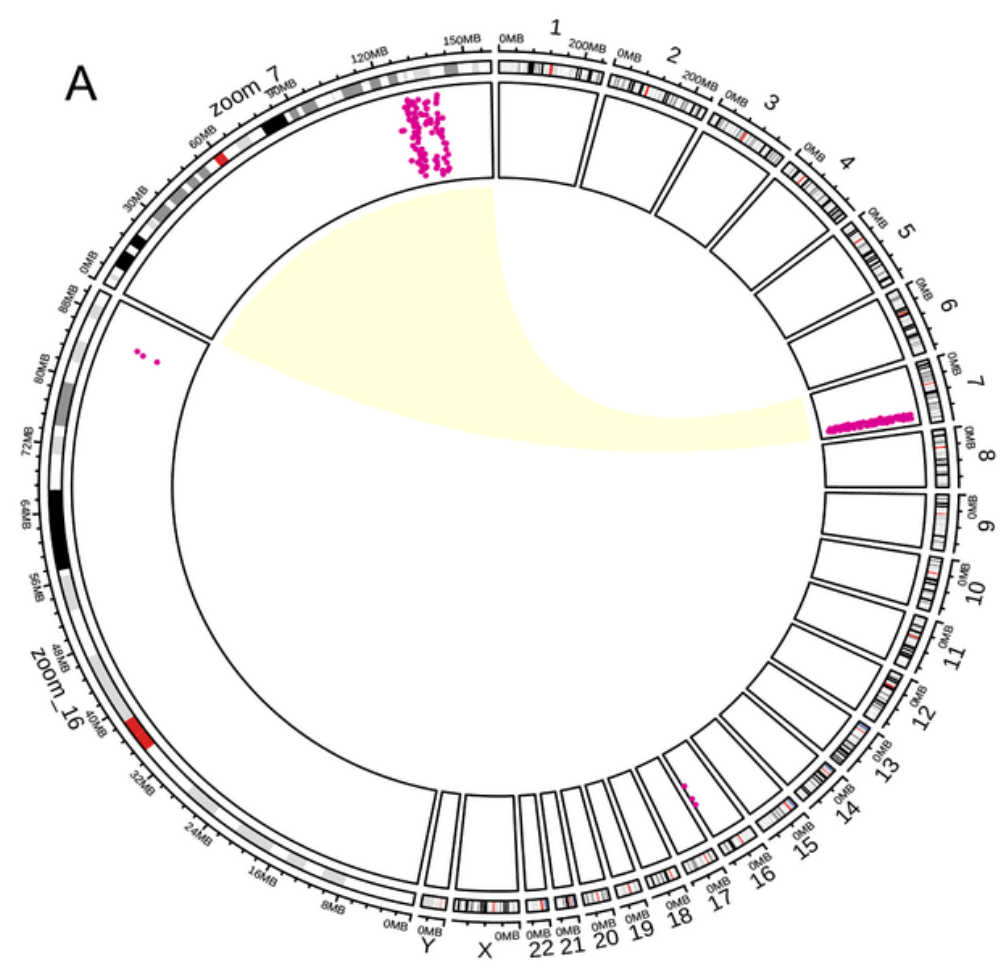

B
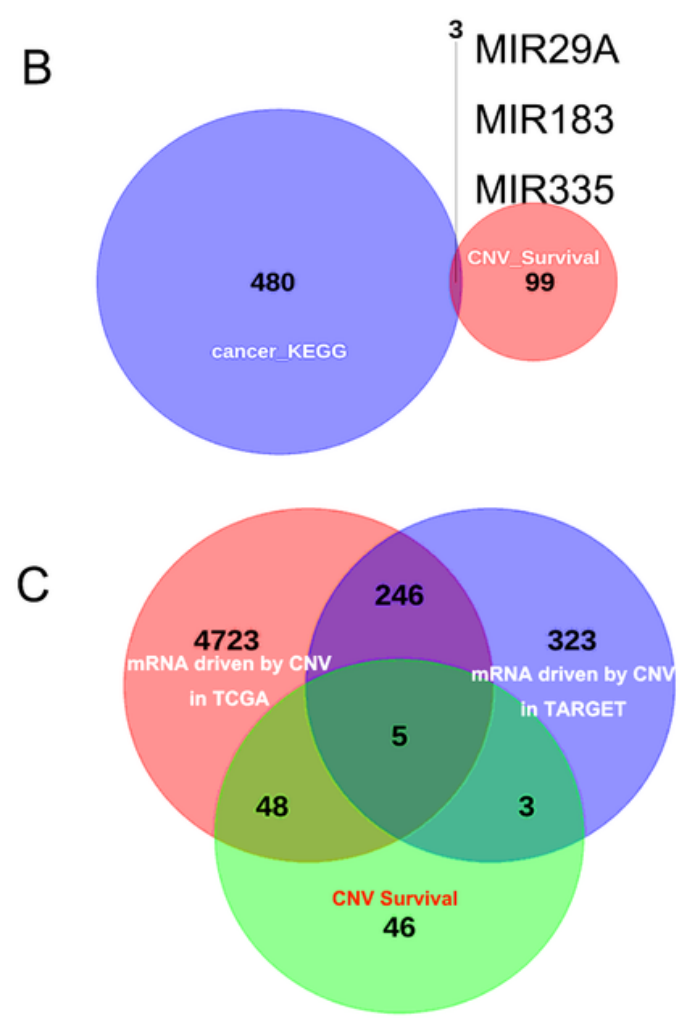

D

+ group=Normal + group=SNV
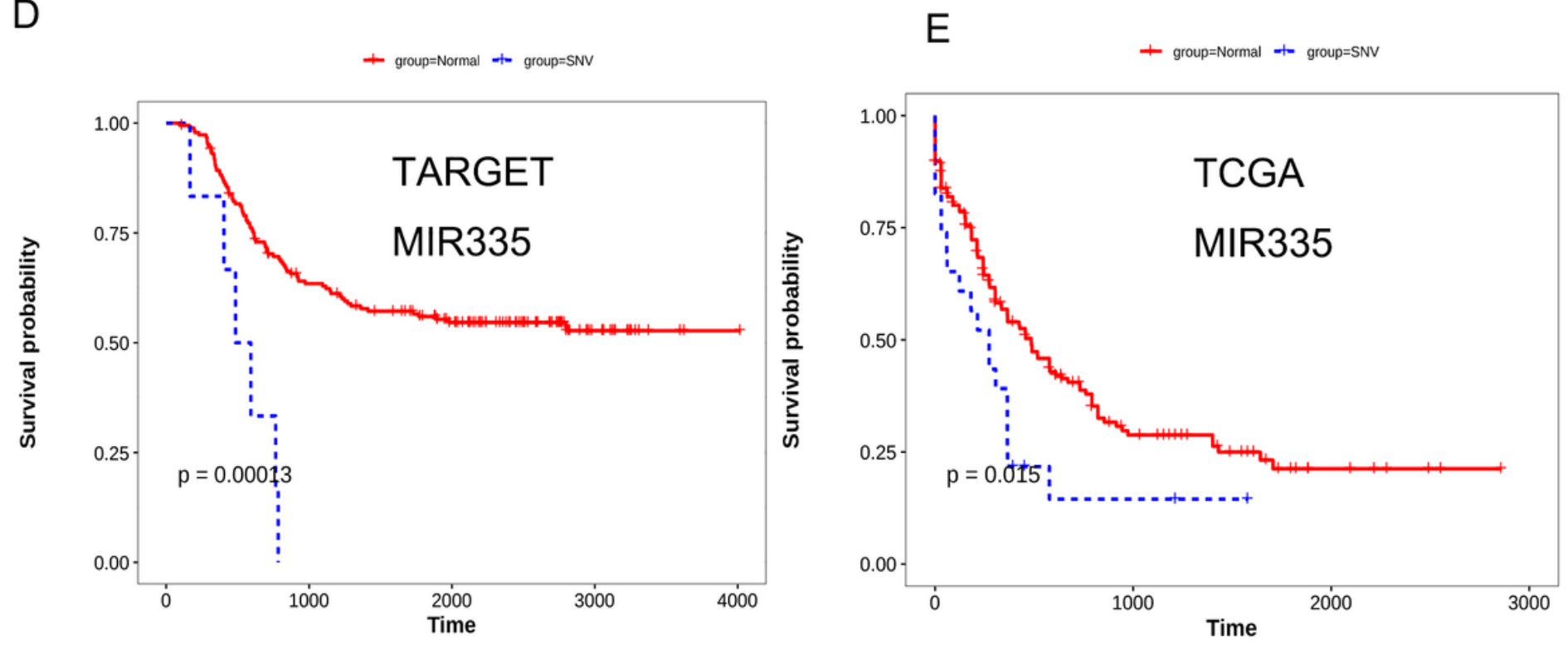

Figure 3

The distribution of CNV genes associated with the survival of AML patients. (b) Zooming diagram showed the chromosome map locations of CNV genes associated with the outcomes of patients. (b) Venn diagram showed the relationship of the CNV genes associated with survival and the KEGG cancer panel. (c) Venn diagram showed the relationship of CNV genes associated with survival and the genes whose expressions were modulated by CNV. $(d, e)$ Kaplan-Meier curve showed the patient survival rates according to the MIR335 gene CNV status. 
A

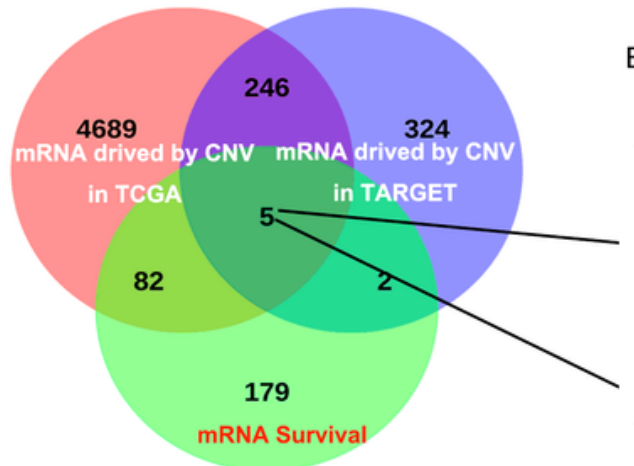

CBFB

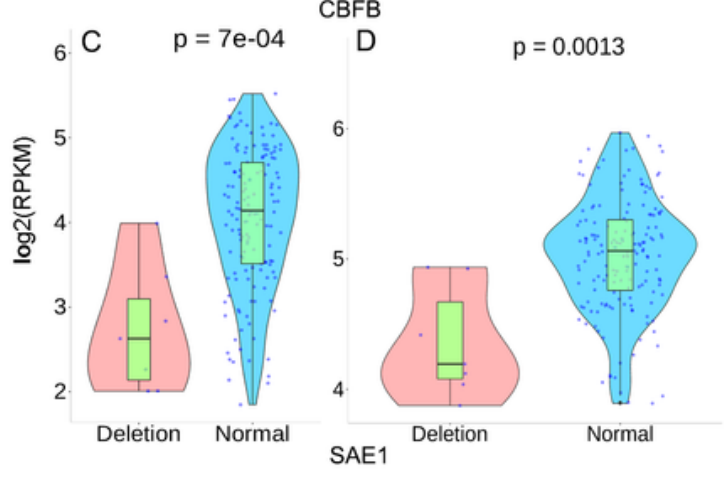

5 E $p=4 e-04 \stackrel{\text { CHAF1B }}{F}$ Kruskal-Wallis, $p=0.0047$

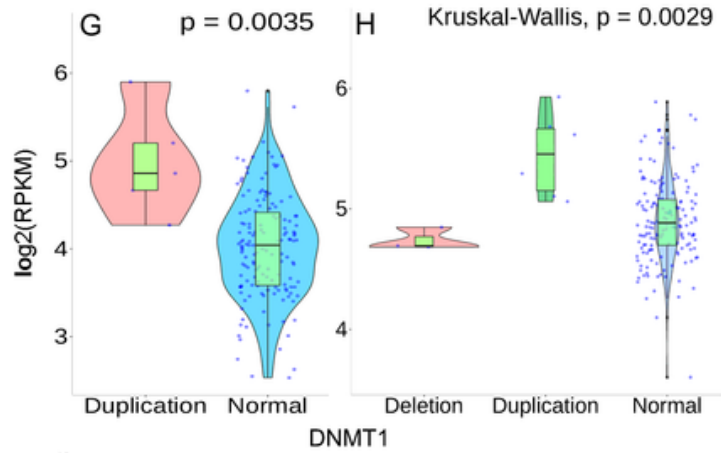

${ }^{6} \mathrm{~K} \quad \mathrm{p}=0.0019^{\mathrm{DNMT}} \mathrm{L} \quad$ Kruskal-Wallis, $\mathrm{p}=0.018$
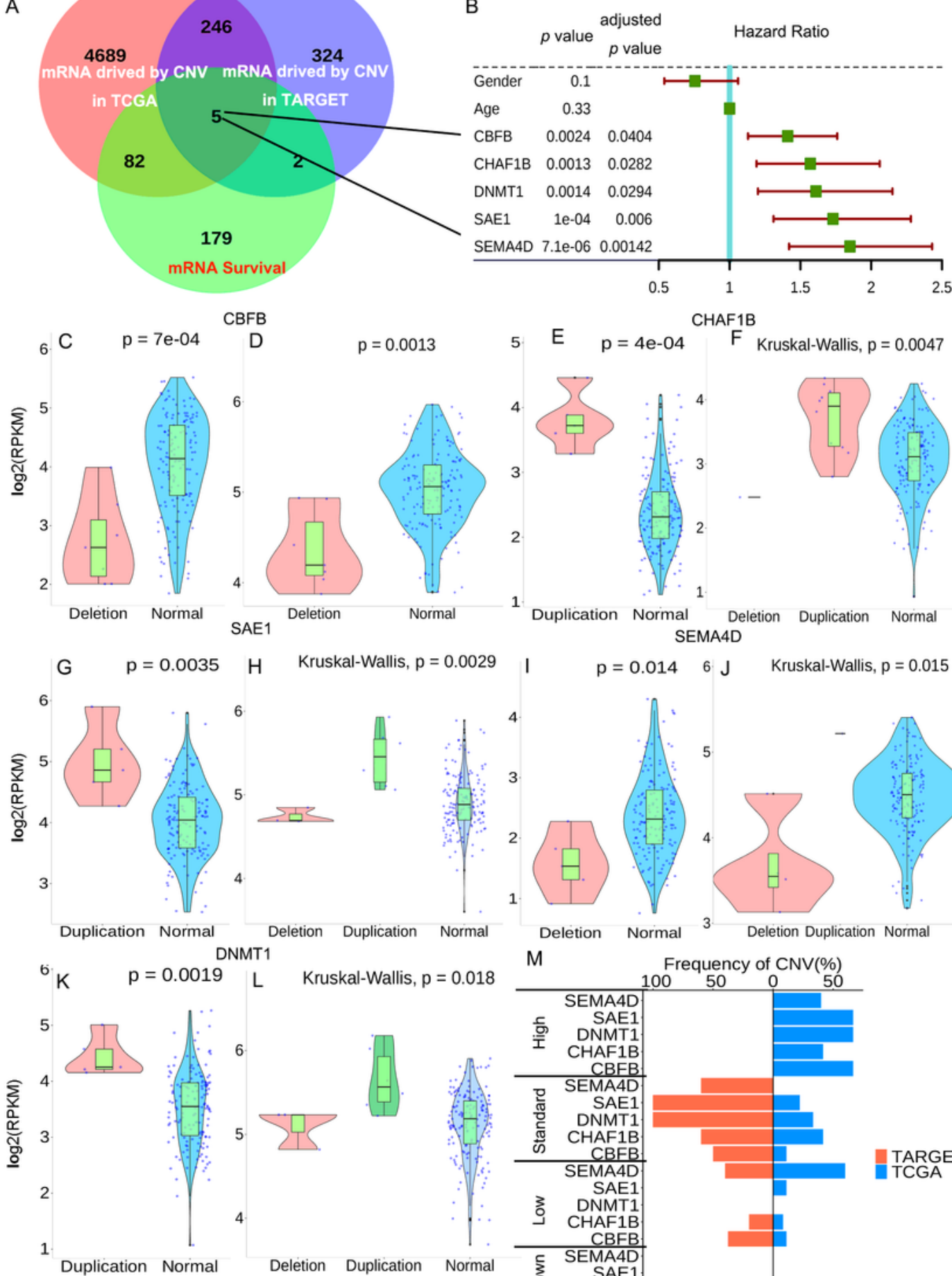

$\begin{array}{lll}\text { CBFB } \quad 0.0024 & 0.0404\end{array}$

$\begin{array}{lll}\text { CHAF1B } & 0.0013 \quad 0.0282\end{array}$

$\begin{array}{lll}\text { DNMT1 } & 0.0014 & 0.0294\end{array}$

SAE1 1e-04 0.006

SEMA4D 7.1e-06 $\quad 0.00142$

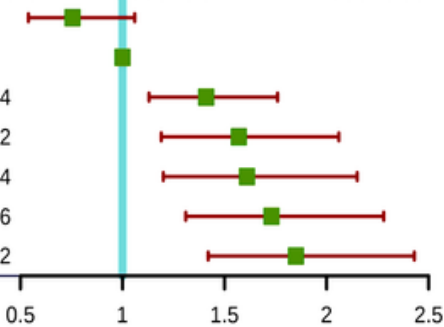


cohort; $d, f, h, j, k$ were the results in the TCGA cohort. (m) The distribution of 5 CNV genes in the risk groups in the two AML cohorts.

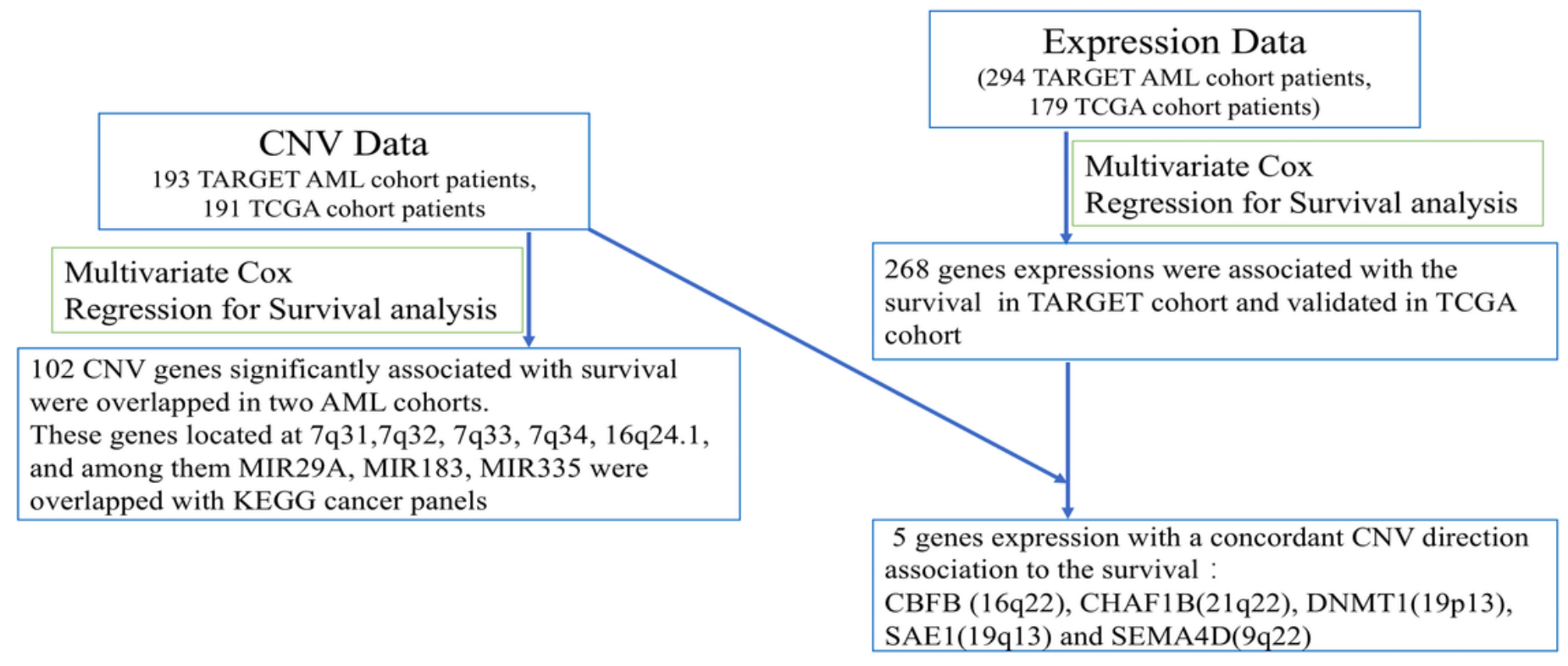

\section{Figure 5}

Pipeline for the discovery of prognostic signature based on the CNV and expression in AML.

\section{Supplementary Files}

This is a list of supplementary files associated with this preprint. Click to download.

- supplementarytable2.docx

- supplementarytable1.docx 